\title{
Secretory phase of menstruation and implantation
}

\author{
Monique Monard*, Courtney Marsh, Katelyn Schumacher and Warren Nothnick \\ Department of Obstetrics and Gynecology, University of Kansas Medical Center, USA
}

\section{Introduction}

The female reproductive system prepares the female body for conception and pregnancy through two distinct cycles, the ovarian cycle and the endometrial cycle. The human endometrium, under the influence of complex biological signals, undergoes cyclic changes in preparation for implantation and the initiation of pregnancy. An array of molecular activity, still poorly understood, gives rise to relatively consistent morphologic changes of the endometrium during each cycle. In an era of assisted reproductive technologies (ART), there exists an ever-increasing demand to delineate these pathways in order to improve pregnancy rates. Ultimately, success in the field of reproduction and fertility requires an understanding of these complex processes, from molecular to cellular to tissue, in both the healthy patient as well as in the setting of various pathologic states.

This article will discuss the endometrial cycle with an emphasis on the secretory phase, including the molecular and biochemical components of endometrial receptivity and implantation. Markers and techniques for assessment of receptivity will be reviewed, as well as pathologic states that alter fertility.

\section{The menstrual cycle and the endometrium}

The endometrium is comprised of two general layers, the functionalis and the basalis. The functionalis is made up of a compact zone, including stroma underneath the luminal epithelium, and a spongy zone which lies above the basalis layer $[1,2]$. The myometrium lies beneath the basalis layer, which undergoes fewer cyclic changes compared to the functionalis layer and is capable of regenerating the functionalis after menstruation (Figure 1).

The endometrial cycle consists of three sequential phases - the proliferative phase, secretory phase, and menstruation. Each phase is marked by physiologic changes that are controlled by circulating levels of estrogen and progesterone, which are influenced in turn by the ovary. The ovarian cycle is characterized by follicular development mediated by FSH (follicular phase), ovulation mediated by the LH surge, and development of the corpus luteum and production of progesterone (luteal phase). In the ideal 28-day cycle, ovulation occurs on Day 14, the corpus luteum becomes atretic on Day 28 and menses begins the next day, Day 1. After ovulation, the remnant of the dominant follicle becomes the corpus luteum, a temporary endocrine structure which produces progesterone (Figure 1) [1-4].

During the proliferative phase, estrogen drives the restoration of the functionalis layer with re-epithelialization by approximately Day 5 of the menstrual cycle. This phase is characterized by hypertrophy and proliferation of glands, increase in stromal matrix, and elongation of terminal arterioles to the endometrial lumen. Estrogen also upregulates the progesterone receptors that orchestrate the environment during the secretory phase, which will be covered in more detail in the next section

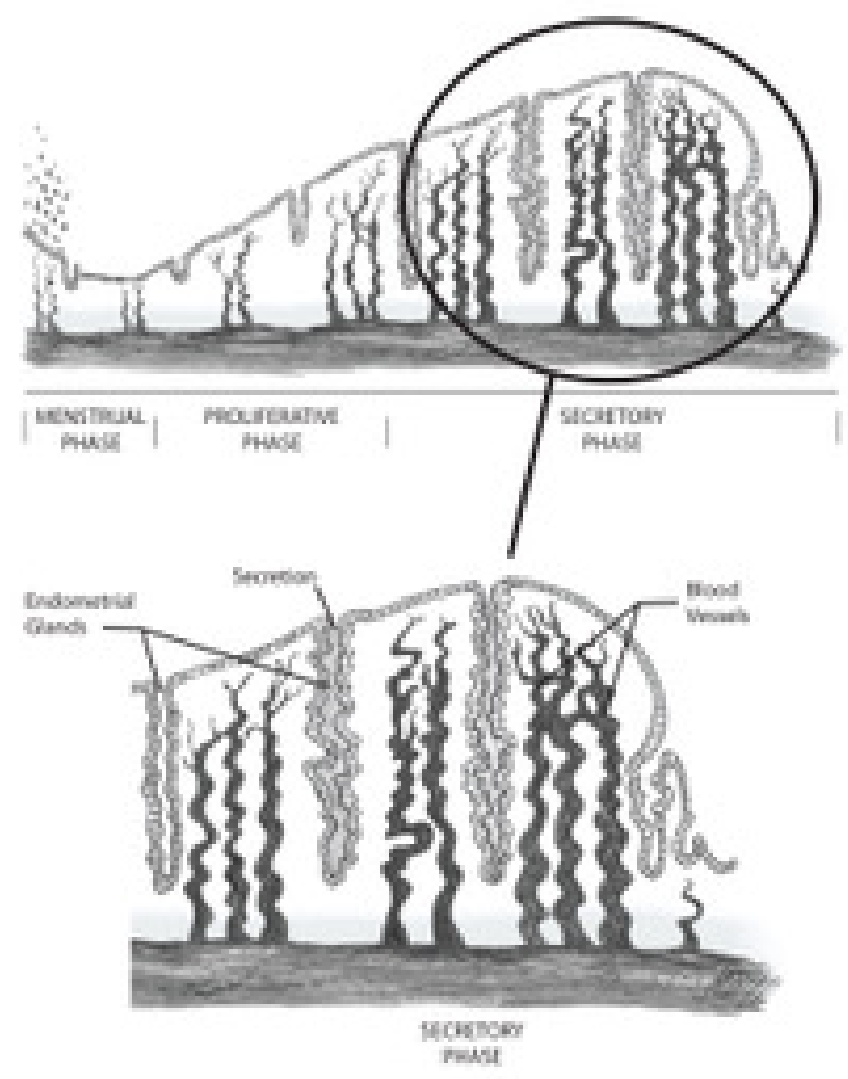

Figure 1. Functional anatomy of the human endometrium during the secretory phase

$[2,4]$. In the absence of fertilization, progesterone levels decline due to atresia of the corpus luteum. This leads to vascular changes, subsequent tissue death and endometrial shedding, or menses.

\section{The secretory phase}

For decades, endometrial dating has been performed histologically $[4,5]$. After ovulation, there is an increase in superficial stromal edema that becomes generalized by Day 21 . Stromal cells near terminal spiral arteries show an increase in cytoplasm and surrounding extracellular matrix, a process termed predecidualization, which eventually encompasses the majority of the superficial endometrium by Day 25 .

${ }^{\star}$ Correspondence to: Monique Monard, Department of Obstetrics and Gynecology, University of Kansas Medical Center, 3901 Rainbow Boulevard, Kansas City, KS 66160, USA, Tel: (954) 629-3935; E-mail: Mmonard@kumc.edu

Received: September 20, 2018; Accepted: October 09, 2018; Published: October 15,2018 
By Day 27, the superficial stromal cells are nearly indistinguishable from decidual cells of pregnancy. The increasing edema during the secretory phase results in the global endometrial thickening that is readily apparent with ultrasonography. Throughout the secretory phase, there are also distinct changes in spiral arteries. They rapidly lengthen, outpacing endometrial thickening, and become increasing coiled [4-7].

During the proliferative phase, there is an increase in glandular epithelium mitotic activity and pseudo stratification of nuclei. There is a parallel increase in the proliferation of stromal components as well during this phase. After ovulation, this process is replaced by secretory transformation of glands and a slowing of stromal proliferation. At the cellular level of glandular epithelial cells, the early-secretory phase is characterized by abundant endoplasmic reticulum, accumulation of glycogen-rich vacuoles, and displacement of nuclei centrally. Six days after ovulation, loss of vacuoles from the cytoplasm corresponds with maximal glandular secretory activity [4,7].

A complex interplay between stromal cells and immune cells highlights the secretory phase of the superficial endometrium. A substantial portion of the uterine leukocyte population is made up of CD56-/CD16+ uterine natural killer (uNK) cells which are believed to play a role in maternal allorecognition of fetal trophoblasts rather than in cytotoxicity. The uNK cells are largely found in proximity to spiral arteries and extravillous trophoblasts in early pregnancy. The cell population increases dramatically after ovulation and vanishes before menses in the absence of pregnancy. The activity of uNK cells have been shown to be controlled by regional steroid hormones as well as local chemokines, including those containing the (C-X-C motif) and various interleukins $[1,4]$.

Macrophages (CD163+) are also found in the superficial endometrium, rapidly accumulating in the stroma after ovulation and declining in the absence of progesterone. $\mathrm{T}$ cells are found scattered throughout the endometrium with little to no cell-cycle change in concentration, as well as uterine dendritic cells (uDCs) which are more prominent in decidua of pregnancy [4].

The end of the secretory phase and beginning of the premenstrual phase is characterized by degradation of the stromal network, infiltration of the stroma by leukocytes, and cessation of glandular activity in the absence of the appropriate signals from hCG. The degradation of the stromal network is catalyzed by matrix metalloproteinases (MMP), which become activated in the setting of reduced progesterone. Evidence of apoptosis is evident throughout the tissue, and the dense internal structural changes in glandular cells characteristic of secretion vanish $[1,4]$.

\section{Microarchitecture and implantation}

The implantation of the blastocyst is a highly organized, selective process that, with some variations, is preserved among many species. It revolves around a "window of implantation" (WOI), during which the endometrium is capable of "receiving" the trophectoderm of the blastocyst. The interaction is orchestrated by a variety of molecules and is overall regulated by steroid hormones [4,7]. More recent research suggests the WOI lasts between 12 hours and 2 days and is often shifted in patients with infertility [3].

In 1950, Noyes et al. conducted histologic examination of early human implantation and observed no change between expected nonpregnant mid-secretory phase endometrium, with the exception of invading trophoblasts displacing adjacent glandular structures [5]. In 1999, Wilcox et al. narrowed down the timing of implantation to 8 to 10 days after ovulation, with increased losses correlated with later implantation [8].

The process of implantation follows several well-described steps, which is coordinated with endometrial preparation by changes in steroid hormones. The focus here starts with apposition and transient attachment of the syncytial trophoblast cells to the endometrium epithelium, which is followed by firm attachment and invasion. As the blastocyst enters the uterine cavity, the zona pellucida is shed allowing for exposure of the trophectoderm [7,9]. Driven by progesterone, the secretory phase endometrial epithelial cells enter a hypersecretory state, with ultrastructural changes described as the Arias-Stella reaction. This hypersecretory state of the tissue provides the necessary nutrition essential for embryo survival, as vascular remodeling occurs later [10].

The molecular basis of invasion is of active interest in the field, as failure of these mechanisms is associated with pregnancy loss. Current research suggests that MUC1, a large transmembrane mucin and a barrier to implantation is downregulated and/or removed through the action of surface proteases and decreased expression of progesterone receptors. The loss of MUC1 allows for appropriate apposition and attachment between the embryo and endometrium. Initial transient attachment is mediated by various molecules including selectins and galectins. More firm attachment is mediated by integrins, including $\alpha v \beta 3$ and $\alpha 4 \beta 1$, and CD44 and their associated ligand osteopontin (OPN). Other associated attachment molecules include trophinin, HBEGF, fibronectin, vitronectin, SPP1, laminin, IGFBP1, and the latency associated peptide linked to TGF $\beta[2-4,7,9]$.

Specialized surface macromolecular structures have also been implicated in blastocyst adhesion and invasion. Endometrial epithelium consists of both ciliated and nonciliated, secretory cells, the proportions of which are regulated by estrogen levels. The secretory cells develop transient surface structures in response to progesterone levels, called pinopodes, during the time of maximal receptivity. These structures are involved in pinocytosis and contain various adhesion molecules, including $\alpha \nu \beta 3$ and OPN. The development of pinopodes is dependent upon HOXA-10, a homeobox gene whose expression is vital for endometrial receptivity, regulating both endometrial stromal cell proliferation and epithelial cell morphogenesis [11]. Blocking the expression of HOXA-10 shows a significant reduction in the number of pinopodes. Although some evidence points to pinopodes role in adhesion and invasion of the embryo, their precise function and overall importance is still debated $[2,3]$.

Adhesion via these molecules is far from a static process. Extracellular contact with appropriate ligands transmits signals, through a variety of processes, into intracellular cascades that results in gene transcription and protein expression. The resultant effect includes adhesion, as well as migration, proliferation, and cytoskeletal remodeling. The overall result is invasion of the embryo into a primed endometrium, where is has the potential for growth and development [2-4].

\section{Receptivity markers and clinical applications}

Endometrial dating has drastically changed since the establishment of the Noye's criteria for histologic dating in 1950 [5]. With a more detailed understanding of the biochemical pathways influenced by steroid hormones during the menstrual cycle came new targets to identify endometrial receptivity. Initially, single molecules were 
analyzed. With the advent of microarray technology, vast amounts of molecules can be analyzed at the same time to give a much more complete picture of the endometrial environment $[1,3,4]$.

Cytokines are involved in many processes throughout the menstrual cycle and have been shown to play a critical role in ovulation and implantation. Leukemia inhibitory factor (LIF) is in the IL- 6 family and expression has been demonstrated in the human endometrial epithelium during the mid- to late-secretory phase [11]. In women of proven fertility, endometrial biopsies demonstrated LIF mRNA expression from day 18 to 28 with a peak at day 20 of the menstrual cycle with a 2.2-fold increase in LIF secretion between the proliferative and secretory phase [12]. IL-6, another cytokine expressed in the endometrium shows a regulated temporal pattern throughout the menstrual cycle with the highest detected levels during the luteal phase. IL-6 mRNA levels increase progressively during the mid- to late-secretory phase with a strong expression during the window of implantation. The protein is strongly pronounced in the epithelial and glandular cells. Bone morphogenic protein 2 (BMP2), a member of the TGF- $\beta$ superfamily, is first detected in the stroma surrounding the site of blastocyst attachment during the mid-secretory phase. BMP2 is considered a critical regulator of decidualization due to its role in regulating proliferation and differentiation, as well as its expression during the implantation period [13].

Amniotic fluid contains a significant amount of prolactin (PRL), which is produced by the decidua. It has been determined that endometrial PRL production begins around cycle day 22, and levels rise throughout pregnancy. Similarly, high levels of IGFBP-1 and LEFTY2 are produced by secretory phase endometrium in response to progesterone. Given their abundance and production during the menstrual cycle, these proteins serve as potential markers for endometrial receptivity, although clinic utility is not yet clear [1-4,9].

Prostaglandins (PGs) have been shown to play a crucial role for successful embryo implantation due to their vasoactive properties. The oeneration of PGs is achieved by cytosolic phospholipase $A_{2}$ $\left(\mathrm{CPL}_{2}\right)$ and cyclooxygenase (COX). Studies in female mice lacking $\mathrm{cPLA}_{2}$ or COX-2 enzymes have demonstrated the vital role of PGs in implantation. $\mathrm{PGE}_{2}$ and $\mathrm{PGF}_{2 \alpha}$ expression was detected in human endometrium throughout all stages of the menstrual cycle but was down-regulated during the late-secretory phase [11].

Several integrins have been focused on as possible markers of uterine receptivity and have been noted to undergo alterations in the epithelium and decidua during implantation. The co-expression of $\alpha 1 \beta 1, \alpha v \beta 3$, and $\alpha 4 \beta 1$ marks the period of endometrial receptivity by mediating firm attachment between the embryo and endometrium. The regulated expression of secretory phase integrins suggests that steroid hormones likely play a role in their presence. The discovery of a1 $\beta 1 /$ laminin receptor (VLA-1) expression on secretory phase endometrial epithelium is suggestive of progesterone up-regulating $\alpha 1 \beta 1$ in the endometrium. The firm attachment mediated by integrins is also dependent on integrin-associated ligands. Secreted phosphoprotein 1 (SPP1), also known as osteopontin, is a ligand for $\alpha v \beta 3$ that has shown significant up-regulation in endometrial epithelial cells during the secretory phase. This glycoprotein mediates cellular adhesion and migration during embryo implantation. Calcitonin, a known up-regulator of $\alpha v \beta 3$, is transiently produced in the uterine epithelia during the period of implantation. It functions to promote the outgrowth of trophoblasts on human endometrial epithelial cells and down-regulates E-cadherin expression $[11,14]$.
Another glycoprotein found in the human endometrium is Mucin-1 (MUC1), a factor that interferes with cellular adhesion when highly expressed on the cell surface. MUC1 is likely the first molecule that the embryo encounters on the way to attachment and is thought to repel the blastocyst until the time and place is ideal for implantation. This is confirmed by the apparent down-regulation of MUC1 by progesterone before implantation in the receptive endometrium of mice. The reduced expression facilitates embryo-epithelial interactions by unmasking cell adhesion molecules on the endometrial surface [11]. When measured in humans, MUC1 showed increased expression during the peri-implantation period, contradicting the studies in other species.

Two cytoskeleton-related proteins, stathmin 1 and annexin A2, have observed opposite regulation in the receptive versus prereceptive endometrium. Stathmin 1 is a phosphoprotein and regulates microtubule dynamics during the progression of the cell cycle, specifically at the embryo implantation site. In the human receptive endometrium, it is down-regulated due to the lower levels needed to support decidualization. Annexin A2, recently identified as one of the apical surface molecules in a receptive human endometrium, is involved in cellular transformation, differentiation, regulation of secretory processes, prolactin release, and prostaglandin formation. In human endometrial epithelium, expression of annexin A2 is high in the mid- to late-secretory phase and decreased in the pre-receptive phase. This pattern of expression, along with in vitro studies showing a role in embryo adhesiveness, suggests annexin A2 is vital for implantation [15].

Gene repressor biomarker, BCL6, is measured to detect endometriosis in women with otherwise unexplained infertility. Expression of this protein is associated with inflammation and significantly elevated values are seen in the secretory phase of patients with endometriosis. Data suggests BCL6 is associated with endometrial dysfunction, primarily progesterone resistance, leading to implantation defects and increased IVF failures [16,17].

Ion channels in the endometrium have demonstrated a role in regulating endometrial receptivity and embryo implantation. The volume of electrolyte-containing fluid in the uterine lumen fluctuates throughout the menstrual cycle under the influence of ovarian hormones and is significantly reduced in the mid-secretory phase. This is suggestive of a net fluid absorption across the endometrium during the receptive phase. Cystic fibrosis transmembrane conductance regulator (CFTR) mediates $\mathrm{Cl}^{-}$efflux, which is essential for epithelial fluid secretion. The endometrial epithelium is known to contain CFTR, playing an active role in endometrial $\mathrm{Cl}^{-}$and fluid secretion. The down regulation of CFTR by progesterone during the secretory phase likely contributes to the decrease in volume, which aids in embryo implantation. The epithelial sodium channel $(\mathrm{ENaC})$ is present in the endometrium and provides a driving force for water absorption after establishing a sodium gradient, contributing to the dynamic regulation of uterine luminal fluid. CFTR has an inhibitory effect on $\mathrm{ENaC}$, so the down-regulation of CFTR during the secretory phase enhances the absorptive activity of the endometrial epithelium. $\mathrm{ENaC}$ is up-regulated by progesterone, furthering the absorptive properties of the endometrial epithelium during the secretory phase. Other ion channels such as $\mathrm{K}^{+}$and $\mathrm{Ca}^{2+}$ and ion transporters, SLC4 and SLC26, are emerging as important players in regulating certain processes of embryo implantation [13].

Microarray analysis of endometrial tissue allows for assessment of hundreds to thousands of molecules at once. Genomic and proteomic 
analysis has identified varying levels of genes and proteins implicated in a wide array of activities during decidualization. Assessment of secreted fluids, called secretomics, which largely looks at protein and lipid levels, allows for high-throughput analysis of endometrial secretions during the secretory phase without the need for biopsies. Although our understanding of microarray technology as it related to infertility is still evolving, there are products on the market that are finding clinical utility and will be discussed in more detail later in the chapter $[3,18,19]$.

The human endometrium has a unique proteomic signature during the secretory phase supported by many studies. Receptivity markers are measured in clinical settings to avoid implantation failure and to hopefully provide a more favorable outcome for patients utilizing ART. Although some of the mentioned biomarkers have only recently been discovered as key players in the human receptive endometrium, these discoveries show promise in better understanding the complex interactions throughout the secretory phase and window of implantation.

\section{Pathologic States and the Secretory Phase}

Given the complex nature of endometrial receptivity, it is not surprising that problems occur. Local factors that can negatively impact receptivity and implantation can be broadly grouped into mechanical and inflammatory factors. Local factors include inflammatory states, such as endometriosis, adenomyosis, hydro salpinges and endometritis, and mechanical abnormalities encompass both congenital anomalies and acquired conditions $[2,20,21]$.

Mechanical abnormalities of the uterus such as uterine septae, fibroids, polyps and adhesions result in physical barriers to successful fertilization and implantation. These conditions are linked with recurrent pregnancy loss and infertility and substantial evidence exists that shows surgical correction of these abnormalities may improve outcomes [2,21].

Given the delicate regulation of the menstrual cycle and of implantation biology, inflammatory factors that affect signaling pathways can clearly derail normal physiologic processes. In the setting of local inflammation, progesterone resistance and estrogen receptor dominance have been shown through microarray analysis, which results in impaired implantation. With endometriosis, for instance, there is an increase in inflammatory cytokines including TNF $\alpha$, INF $\gamma$, IL-1 and IL-17. This leads to downstream effects such as phosphorylated STAT3, which in turn helps lead to an estrogen dominant and progesterone resistant state [2,20]. Decreased expression of IL-11 and CCL4, which are associated with embryo receptivity, is found in chronic endometritis and is believed to be related to infertility associated with this condition [22]. Low integrin levels have been associated with inflammatory conditions and reduced $\alpha v \beta 3$ expression, which can be caused by increased estrogen levels, and have been tied to IVF failure. Conversely, aromatase overexpression is seen in inflammatory states, and is linked with predicting failure in ART cycles. Other chemokines and cytokines, such as interleukins, are similarly linked to inflammation and pregnancy failure [2].

Although there still exist many gaps in our understanding of endometrial receptivity and implantation at the level of the uterus, pregnancy and ART failure cannot be fully explained by local factors. There exist many systemic disorders that can impact the uterine environment and the embryo's ability to implant. These factors include thyroid disorders, vitamin $\mathrm{D}$ deficiency, prolactin disorders, inflammatory bowel disease, obesity and smoking. There exists significant evidence relating hypothyroidism and infertility/IVF success rates, prompting levothyroxine treatment in patients with TSH $<2.5 \mathrm{mIU} / \mathrm{L}$ [2]. Obesity has similarly been linked to infertility, with evidence to support weight loss to improve pregnancy rates $[2,23]$. However, our understanding of how these systemic states affects fertility is still limited, and further research is warranted to fully evaluate these relationships.

\section{Future Directions}

Historically, dating of the receptive endometrium has largely been based off of morphologic criteria [5]. Shortcomings in this method, fueled by significant advances in our molecular understanding of the endometrial cycle, have led to new technologies. Although utilization of single molecular signatures has not yielded satisfactory results, highthroughput analysis of looking at a vast array of markers has been more promising [3]. The recent creation of the "-omics", or fields that utilize high-throughput techniques with sophisticated large data analysis to generate far more detailed pictures of molecular and biochemical processes, has revolutionized our understanding of the receptive endometrium $[3,4,18,19]$.

The analysis of the endometrial cycle using transcriptomics, or the study of DNA transcription in various environments, has been under heavy investigation for over a decade [3]. Using microarray gene expression techniques, researchers have been able to study the expression of thousands of genes simultaneously during different phases of the endometrial cycle. They have identified a unique gene profile during the window of implantation, which includes important factors previously identified such as Leukemia inhibitory factor (LIF), Osteopontin, CXCL14, glycodelin, IL15, L-selectin ligands and various antioxidants [3]. Using this transcription signature, Díaz-Gimeno et al. [24] have created a clinical tool to identify endometrial receptivity. In controlled trials, use of this tool, termed the Endometrial Receptivity Array (ERA), has identified shifted windows of implantation in women with infertility. Furthermore, embryo transfer during the appropriate window has yielded pregnancy and implantation rates similar to control groups [25].

Although transcriptomics has yielded impressive results, it relies on a tissue biopsy. Researchers have analyzed endometrial fluid for composition starting back in the 1970s [21]. More recently, technologies allow high-throughput analysis of vaginal secretions. This area of study has been coined secretomics [26,27]. As it focuses on sampling extracellular fluid, molecules of interest are largely proteins and lipids. Protein analysis has largely revealed increased levels of proteins described by other techniques. However, trauma from merely introducing the catheter to obtain a sample has been postulated to correlate with increased levels of structural proteins [18]. Analysis of lipid levels has revealed elevated levels of PGE2 and PGF2 $\alpha$ during the WOI. Although preliminary information of endometrial secretion analysis is intriguing, further investigation is required [18,27].

Our understanding of infertility and endometrial receptivity has come a long way over the past several decades. However, many questions remain unanswered. New information on molecular and biochemical markers during the endometrial cycle continue to be discovered. These include potential targets for pharmaceuticals. These discoveries are fueled by significant advances in research technologies. High-throughput analysis has revolutionized the field. Next generation sequencing, or massively parallel sequencing, will allow an even more detailed look at the unique signature of the receptive endometrium. 
Translation of this research into clinical trials, and then clinical practice, will have a major impact on the field of reproductive endocrinology and infertility.

\section{References}

1. Critchley HO, Saunders PT (2009) Hormone receptor dynamics in a receptive human endometrium. Reprod Sci 16: 191-199. [Crossref]

2. Fox C, Morin S, Jeong JW, Scott RT Jr, Lessey BA (2016) Local and systemic factors and implantation: what is the evidence? Fertil Steril 105: 873-884. [Crossref]

3. Gómez E, Ruíz-Alonso M, Miravet J, Simón C (2015) Human Endometrial Transcriptomics: Implications for Embryonic Implantation. Cold Spring Harb Perspect Med 5: a022996. [Crossref]

4. Gellersen B, Brosens JJ (2014) Cyclic decidualization of the human endometrium in reproductive health and failure. Endocr Rev 35: 851-905. [Crossref]

5. Noyes RW, Hertig AT, Rock J (1950) Dating the endometrial biopsy. Fertil Steril 1: 3-25.

6. Rock J, Bartlett MK (1937) Biopsy studies of human endometrium: criteria of dating and information about amenorrhea, menorrhagia, and time of ovulation. $\mathrm{J} \mathrm{Am} \mathrm{Med}$ Assoc 108: 2022-2028. [Crossref]

7. Bazer FW, Spencer TE, Johnson GA, Burghardt RC, Wu G (2009) Comparative aspects of implantation. Reproduction 138: 195-209. [Crossref]

8. Wilcox AJ, Baird DD, Weinberg CR (1999) Time of implantation of the conceptus and loss of pregnancy. N Engl J Med 340: 1796-1799. [Crossref]

9. Bazer FW, Wu G, Spencer TE, Johnson GA, Burghardt RC, et al. (2010) Novel pathways for implantation and establishment and maintenance of pregnancy in mammals. Mol Hum Reprod 16: 135-152. [Crossref]

10. Arias-Stella J (2002) The Arias-Stella reaction: facts and fancies four decades after. $A d v$ Anat Pathol 9: 12-23. [Crossref]

11. Achache H, Revel A (2006) Endometrial receptivity markers, the journey to successful embryo implantation. Hum Reprod Update 12: 731-746. [Crossref]

12. Garrido-Gómez T, Quiñonero A, Antúnez O, Díaz-Gimeno P, Bellver J, et al. (2014) Deciphering the proteomic signature of human endometrial receptivity. Hum Reprod 29: 1957-1967. [Crossref]

13. Ruan YC, Chen H, Chan HC (2014) Ion channels in the endometrium: regulation of endometrial receptivity and embryo implantation. Hum Reprod Update 20: 517-529. [Crossref]
14. Lessey BA (1998) Endometrial integrins and the establishment of uterine receptivity. Hum Reprod 13: 247-258. [Crossref]

15. Dominguez F, Garrido-Gomez T, Lopez JA, Camafeita E, Quinonero A, et al. (2009) Proteomic analysis of the human receptive versus non-receptive endometrium using differential in-gel electrophoresis and MALDI-MS unveils stathmin 1 and annexin A2 as differentially regulated. Hum Reprod 24: 2607-2617. [Crossref]

16. Evans-Hoeker E, Lessey BA, Jeong JW, Savaris RF, Palomino WA, et al. (2016) Endometrial BCL6 Overexpression in Eutopic Endometrium of Women with Endometriosis. Reprod Sci 23: 1234-1241. [Crossref]

17. Miravet-Valenciano JA, Rincon-Bertolin A, Vilella F, Simon C (2015) Understanding and improving endometrial receptivity. Curr Opin Obstet Gynecol 27: 187-192. [Crossref]

18. Berlanga O, Bradshaw HB, Vilella-Mitjana F, Garrido-Gómez T, Simón C (2011) How endometrial secretomics can help in predicting implantation. Placenta 3: S271-S275. [Crossref]

19. Díaz-Gimeno P, Ruíz-Alonso M, Blesa D, Simón C (2014) Transcriptomics of the human endometrium. Int J Dev Biol 58: 127-137. [Crossref]

20. Ahn SH, Singh V, Tayade C (2017) Biomarkers in endometriosis: challenges and opportunities. Fertil Steril 107: 523-532. [Crossref]

21. Revel A (2012) Defective endometrial receptivity. Fertil Steril 97: 1028-1032. [Crossref]

22. Kitaya K, Matsubayashi H, Yamaguchi K, Nishiyama R, Takaya Y, et al. (2016) Chronic Endometritis: Potential Cause of Infertility and Obstetric and Neonatal Complications. Am J Reprod Immunol 75: 13-22. [Crossref]

23. Marsh CA, Hecker E (2014) Maternal obesity and adverse reproductive outcomes: reducing the risk. Obstet Gynecol Surv 69: 622-628. [Crossref]

24. Wolf DP, Mastroianni L Jr (1975) Protein composition of human uterine fluid. Fertil Steril 26: 240-247. [Crossref]

25. Díaz-Gimeno P, Horcajadas JA, Martínez-Conejero JA, Esteban FJ, Alamá P, et al (2011) A genomic diagnostic tool for human endometrial receptivity based on the transcriptomic signature. Fertil Steril 95: 50-60. [Crossref]

26. Hood BL, Liu B, Alkhas A, Shoji Y, Challa R, et al. (2015) Proteomics of the human endometrial glandular epithelium and stroma from the proliferative and secretory phases of the menstrual cycle. Biol Reprod 92: 106. [Crossref]

27. Cheong Y, Boomsma C, Heijnen C, Macklon N (2013) Uterine secretomics: a window on the maternal-embryo interface. Fertil Steril 99: 1093-1099. [Crossref]

Copyright: $(2018$ Monard M. This is an open-access article distributed under the terms of the Creative Commons Attribution License, which permits unrestricted use, distribution, and reproduction in any medium, provided the original author and source are credited. 